\title{
Relaxation phenomena in dense, glassy membranes prepared from a polymer solution, monitored by changes in dielectric properties
}

\author{
R.H.B. Bouma ${ }^{a}$, Th. van den Boomgaard ${ }^{a}{ }^{*}$, C.A. Smolders ${ }^{a}$, H. Strathmann ${ }^{\text {a }}$, \\ P.F. Mijnlieff ${ }^{b}$ \\ " Department of Chemical Technology, University of Twente, P.O. Box 217, 7500 AE Enschede, Netherlands \\ ${ }^{\mathrm{h}}$ Department of Applied Physics, University of Twente, P.O. Box 217, 7500 AE Enschede, Netherlands
}

Accepted 7 February 1995

\begin{abstract}
The drying of a solvent-cast polymer film is monitored in a non-invasive way, by measuring the changes in time of dielectric properties, using interdigitated or comb electrodes. Experimentally, the vitrification of the polymer solution is observed at a distinct time, followed by the slow evaporation of solvent from the glassy state. As the solvent diffusion coefficient is a strong function of the concentration in the polymer film, removal of residual solvent proceeds at a self-decreasing rate. With a simple model, solvent mass transfer coefficients in the glassy state are determined from the experiments. It is shown that volume relaxation may be slow compared to the drying process, when preparing thin solvent-cast membranes and using solvents that diffuse relatively fast through the glassy polymer film.
\end{abstract}

Keywords: Dense membranes; Glassy polymers; Dielectric constant; Solvent evaporation; Vitrification

\section{Introduction}

For materials and especially polymers in the glassy state, the momentary configuration can strongly depend on its thermal history. If a sample is quenched from a temperature above the glass transition, excess free volume is frozen into the structure. Physical aging, i.e. the relaxation towards the equilibrium free volume, proceeds at a self-decreasing rate [1]. Therefore material properties will depend on the thermal history of the material.

One can also enter the glassy state from a solution, by extracting or evaporating the solvent. Instead of heat transport, mass transfer is involved in the solidification process. The latter process in general takes more time

\footnotetext{
* Corresponding author.
}

for solidification. But also in this case the properties of the material may depend on its history [2-4]. During the drying process the timescale of volume relaxation may become larger than the timescale of diffusion [5]. It implies that excess free volume will be frozen into the polymeric matrix when volume relaxation is very slow and this is expected to occur upon drying in thin solvent-cast films. The excess free volume will disappear then by physical aging. It has been found in permeation of inert gases through thin films of glassy polymers, in which the accesible free volume is an important parameter, that gas fluxes decrease in time [6].

The material property of interest in this paper is the complex dielectric constant. Dielectric spectroscopy is a versatile technique to determine the polarization of 
dipoles in an applied electric field. The permanent or induced dipole moments in a molecule will change their orientations upon the interaction with the electric field. Therefore, a small disturbance of the equilibrium configuration of a material is needed to measure an intrinsic property of the material.

The solidification of a drying polymer solution will be investigated. There the relatively low frequencies are of interest when monitoring the mobility of the dipoles. The solvent molecules will respond faster to changes in the electric field compared to the polymer.

Usually the material of interest is inserted into a condenser or capacitor and its capacitance can be measured from the response in an electric network. In order to follow continuously the change in dielectric properties of a drying polymer solution a special electrode configuration is needed. Hill et al. use two metal strips on glass or wood substrates as the electrodes $[7,8]$. These configurations suffer, however, from the inability to calculate the dielectric constant from the measured capacitance, because the potential distribution between the electrodes is unknown.

With the appearance of interdigitated or comb electrodes [9], fabricated onto an insulating substrate, dielectric permittivities and loss factors could be measured. Each electrode has a comb-like shape and interdigitates the other. Due to its ease of fabrication, and the quantative measurements possible, this configuration has been adopted in our experiments.

The development of the microdielectrometer sensor [9-11] is also based on the electrode configuration of the comb-electrodes and is essentially a field effect transistor.

In the present paper the drying of a solvent-cast polymer film is monitored by measuring changes in dielectric properties. A model is proposed for solvent transport in the glassy state of the film and solvent mass transfer coefficients are calculated.

\section{Theory}

The polarization $\boldsymbol{P}$ of a material is defined as the sum of the dipole moment vectors per unit volume. The permanent or induced dipoles try to oppose the applied electric field. Because the alignment of the dipoles is not instantaneous, the polarization lags behind in an alternating electric field. A linear, time-invariant, the- ory of electric polarization, i.e. the equivalent of linear viscoelasticity, has been developed to describe this phenomenon.

Assuming an isotropic material, the electric displacement $\boldsymbol{D}(t)$ can be written, according to the Boltzmann superposition principle [12], as a function of the complete history of the electric field $\boldsymbol{E}(t)$.

$D(t)=\int_{-\infty}^{t} g\left(t-t^{\prime}\right) E\left(t^{\prime}\right) \mathrm{d} t^{\prime}$

with $g(t)$ being a retardation memory function, describing the response of the electric displacement in time to a unit pulse in the electric field, and $t^{\prime}$ the time variable of integration.

One can extend the integral in Eq. (1) to infinity by stating that future events do not contribute to the present. Then the integral represents a convolution, and with the help of the Fourier transformation a simple relationship is obtained between the Fourier transforms of electric displacement and electric field.

$D(i \omega)=\epsilon_{0} \epsilon^{*}(i \omega) E(i \omega)$

where $\epsilon_{0} \epsilon^{*}(i \omega)$ is the Fourier transform of $g(t), \epsilon_{0}$ is the dielectric permittivity of free space, i.e. $8.8542 \cdot 10^{-12} \mathrm{Fm}^{-1}$, and $\epsilon^{*}(i \omega)$ is the relative, complex dielectric constant, defined as

$\epsilon^{*}(i \omega)=\epsilon^{\prime}(\omega)-i \epsilon^{\prime \prime}(\omega)$

with $\epsilon^{\prime}(\omega)$ being the relative dielectric permittivity, $\epsilon^{\prime \prime}(\omega)$ the relative loss factor and $i$ the imaginary unit. Throughout this paper the adjective "relative" is usually omitted. The loss factor accounts for the dielectric loss due to the polarization of dipoles and the conductivity due to the presence of free ions.

$\epsilon^{\prime \prime}(\omega)=\epsilon_{\text {polarization }}^{\prime \prime}(\omega)+\frac{\sigma_{\mathrm{d} c}}{\omega \epsilon_{0}}$

where $\epsilon_{\text {polarization }}^{\prime \prime}(\omega)$ is the dielectric loss due to polarization and $\sigma_{\mathrm{d} c}$ the direct current conductivity.

Performing experiments with harmonic signals, the ratio of amplitudes of the electric displacement and the electric field will give the magnitude of the complex dielectric constant, and the phase difference between the two will give the phase of the complex dielectric constant.

In dielectric experiments often a harmonic voltage or potential $\Delta \mathrm{V}$ is applied to the electrodes of a capac- 
itor and an electric field is created in the material between the electrodes. The response of the material, the electric displacement, follows from Eq. (2) and is related to the current $I$ going to the electrode configuration. The complex electric impedance $Z(i \omega)$ of a capacitor with a complex capacitance $C^{*}(i \omega)$, for example the comb-electrodes, is defined by the complex ratio of applied voltage and current.

$Z(i \omega) \equiv \frac{\Delta V(i \omega)}{I(i \omega)}=\frac{1}{i \omega C^{*}\left(\epsilon^{*}(i \omega)\right)}$

The relation between the voltage applied to and the current going through the electrode configuration is a measure of the dielectric properties of the material between the electrodes, the configuration of the electrodes and possible resistances in the electrodes.

In the experiments the dielectric properties of a polymer solution that is changing during the experiment in a very thin polymeric layer, will be investigated. The change of dielectric properties with time is monitored at a fixed frequency of the voltage applied to the combelectrodes.

\section{The experimental set-up}

In the experiments the dielectric properties of a polymer solution, that is changing during the experiment into a very thin polymeric layer, will be investigated.

Because of its ease of fabrication the comb-electrode configuration, which makes a quantitative measurement of dielectric properties possible, has been chosen for the experiments. Since the tests should be carried out with very thin polymer films of less than $20 \mu \mathrm{m}$ thickness a restriction is placed onto the inter-electrode spacing.

Comb-electrodes have been made by selectively etching the chromium from a chromium mask with a photolithographic technique. The thickness of the chromium layer is about $100 \mathrm{~nm}$. The inter-electrode distance and the width of the electrodes are approximately $5 \mu \mathrm{m}$. The surface is $1.4 \mathrm{~cm}^{2}$. The minimal thickness of the polymeric layer covering the electrodes, with an electrode spacing of $5 \mu \mathrm{m}$, should be about $7 \mu \mathrm{m}$. This layer of $7 \mu \mathrm{m}$ contributes to the observed complex capacitance of the comb-electrodes assuming that the charge density in the polymeric layer and glass support is zero and that the finite thickness of the electrodes

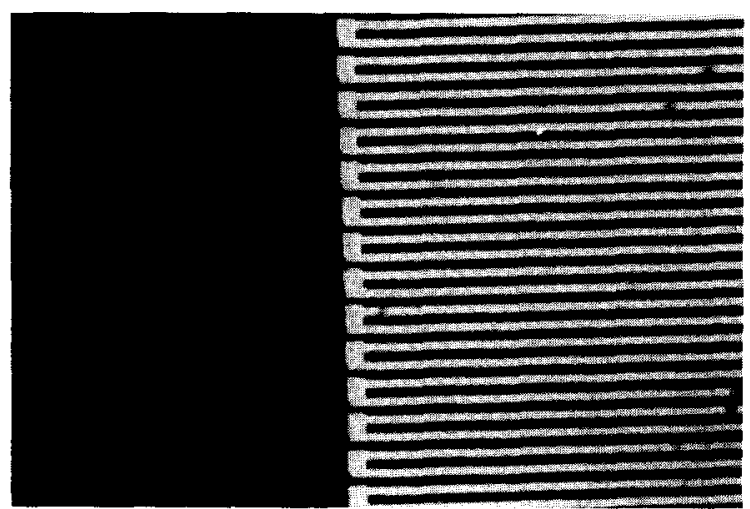

Fig. 1. Top view of the comb-electrode configuration as seen with a light microscope.

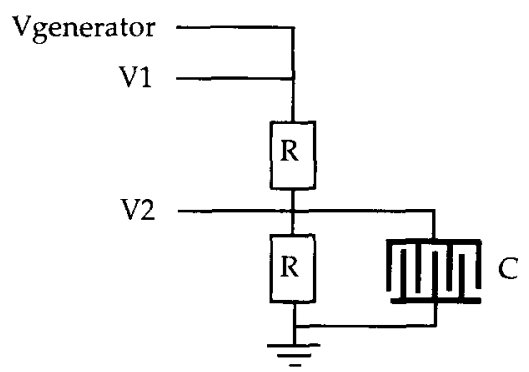

Fig. 2. Schematic diagram illustraing the electric network which was used in this study. The electric network consists of two resistors $R$ and a non-ideal capacitor $C$, the comb-electrodes. V1 and V2 are the two measured voltages.

may be neglected. In Fig. 1 part of the comb-electrodes is shown, as observed with a light microscope.

The comb-electrode configuration is prepared on a glass surface. This means that not only the polymer solution or polymer film will contribute to the measured capacitance, but also the glass support. The respective contributions to the capacitance are acting parallel and may therefore be added. Neglecting the resistances in the electrodes and assuming the same proportion of the dielectric properties to their respective capacitances, based on the small thickness of the comb-electrodes compared to the electrode spacing, one may write:

$C^{*}(i \omega)=k \epsilon_{0}\left\{\epsilon_{\text {glass }}^{*}(i \omega)+\epsilon_{\text {polymer }}^{*}(i \omega)\right\}$

with $k$ being a geometry factor with unit length.

In Fig. 2 the electric network, in which the combelectrode configuration is incorporated, is drawn.

The complex ratio of the two measured voltages $V_{1}$ and $V_{2}$ is called a transfer function and is given by: 


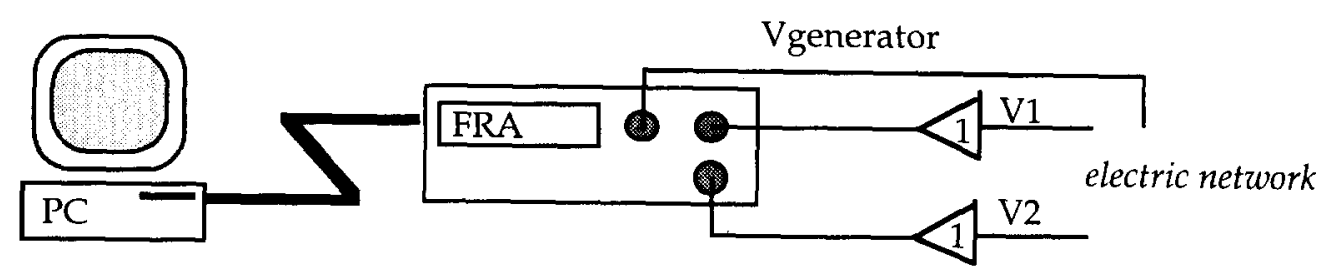

Fig. 3. Automated experimental set-up for the determination of the complex dielectric constant. The electric network is given in Fig. 2.

$\frac{\mathrm{V}_{1}}{\mathrm{~V}_{2}}=2+i \omega R C^{*}(i \omega)$

Here $i$ is the imaginary unit, $\omega$ the angular frequency of the sinusoidal voltage applied by the generator, $R$ the known resistance of the two resistors in the electric network and $C^{*}$ the complex capacitance of the combelectrodes.

The contribution of the glass substrate to the measured capacitance can be eliminated, when the resistance in the electrodes may be neglected, by measuring first the transfer function with the polymeric material of interest, indicated by subscript $\mathrm{p}$, and then measuring the same transfer function of the bare comb-electrodes in a nitrogen atmosphere, indicated by subscript $\mathrm{N}_{2}$, and subtracting these two values at corresponding frequencies. This will give relation (8).

$$
\begin{aligned}
\left(\frac{\mathrm{V}_{1}}{\mathrm{~V}_{2}}\right)_{\mathrm{p}}-\left(\frac{\mathrm{V}_{1}}{\mathrm{~V}_{2}}\right)_{\mathrm{N}_{2}}= & \omega R k \epsilon_{0} \epsilon_{\mathrm{p}}^{\prime \prime} \\
& +i \omega R k \epsilon_{0}\left(\epsilon_{\mathrm{p}}^{\prime}-\epsilon_{\mathrm{N}_{2}}^{\prime}\right)
\end{aligned}
$$

Calibration of the comb-electrodes according to Eq. (8) has been performed with the Baysilon M350 polydimethylsiloxane oil from Bayer, supplied by Necarbo. The geometry factor $k$ is $10.0 \pm 0.4 \mathrm{~m}$. The relatively large error in the calibration factor results from the limited accuracy of the electronic equipment.

Because of the thin chromium layer the resistance in the electrodes may be of the same order as the impedance between the two electrodes. Evaluation of experimental data with Eq. (8) is valid only when the absolute value of the impedance due to the complex capacitance of the glass support and polymer film is much larger than the total resistance of the chromium electrodes, i.e.

$|Z(i \omega)|=\frac{1}{\omega\left|C^{*}(i \omega)\right|} \gg 2 \frac{\rho L}{A}$ with $\rho$ the resistivity of chromium $\left(13 \cdot 10^{-8} \Omega \mathrm{m}\right), L$ the length of a needle of an electrode $\left(1.4 \cdot 10^{-2} \mathrm{~m}\right)$ and $A$ the total cross-section of one comb perpendicular to the direction of the current in the electrodes $\left(3.5 \cdot 10^{-10} \mathrm{~m}^{2}\right)$. The area $A$ corresponds to a chromium layer thickness of $100 \mathrm{~nm}$, an electrode width of $5 \mu \mathrm{m}$ and 700 needles per comb. The total resistance in the electrodes is $10.4 \Omega$. Therefore, no measurements are performed at frequencies exceeding $10000 \mathrm{~Hz}$ and only the final part of the drying curves is plotted.

An automated experimental set-up has been designed for generating the input signal of the electric network in Fig. 2, measuring the transfer function and collecting the data (see Fig. 3). A personal computer controls via an IEEE-bus the Solartron 1255 frequency response analyzer (FRA). Two low frequency, high impedance, buffers [13] have been constructed to match the high impedances of the electric network and the relatively low input impedances of the FRA $\left(10^{6}\right.$ $\Omega$ in parallel with $35 \mathrm{pF}$ ). The buffers, indicated by the amplifier symbol and amplification factor 1 , are constructed with the help of operational amplifiers with an input impedance of $10^{12} \Omega$ in parallel with $3 \mathrm{pF}$. The output impedance of each buffer is $50 \Omega$.

The comb-electrodes are placed in a box in which the atmosphere is controlled by a continuous flow of nitrogen.

\section{Results}

With the comb-electrodes one can monitor the drying of a polymer solution in a non-invasive way. In the experiments amorphous polymers have been dissolved in a suitable solvent which is evaporating into a nitrogen atmosphere upon casting the solution on the combelectrodes.

The polymers chosen are polyacrylonitrile (Aldrich), polyethersulfone E1000 (BASF), and the 
Table 1

Dielectric properties, as supplied by the manufacturers, of polymers used in the dielectric experiments

\begin{tabular}{|c|c|c|c|}
\hline $\begin{array}{l}\text { Frequency } \\
(\mathrm{Hz})\end{array}$ & $\begin{array}{l}\text { Temperature } \\
\left({ }^{\circ} \mathrm{C}\right)\end{array}$ & $\begin{array}{l}\text { Dielectric } \\
\text { permittivity }\end{array}$ & Dielectric loss \\
\hline \multicolumn{4}{|c|}{ Polyethersulfone E $1000^{a}$} \\
\hline 50 & 23 & 3.6 & $1.7 \cdot 10^{-3}$ \\
\hline $1 \cdot 10^{\circ}$ & 23 & 3.5 & $11 \cdot 10^{-3}$ \\
\hline \multicolumn{4}{|c|}{ Polyimide Matrimid 5218 } \\
\hline $1 \cdot 10^{2}$ & 25 & 3.4 & \\
\hline $1 \cdot 10^{3}$ & 25 & 3.3 & $8.5 \cdot 10^{-3}$ \\
\hline $1 \cdot 10^{6}$ & 25 & 3.0 & \\
\hline $1 \cdot 10^{3}$ & 60 & 3.0 & $11.7 \cdot 10^{-3}$ \\
\hline $1 \cdot 10^{3}$ & 100 & 3.0 & \\
\hline $1 \cdot 10^{3}$ & 150 & 3.0 & \\
\hline
\end{tabular}

Moisture might be present in the polymer sample due to the humidity of air.

${ }^{b} \mathrm{XU} 218$ is the experimental version of Matrimid 5218.

polyimide XU 218 (Ciba-Geigy). Dielectric data are given in Table 1.

The polyimide dielectric permittivities are given at four different temperatures. The measured permittivity at $25^{\circ} \mathrm{C}$ is high compared to the permittivities at 60 , 100 and $150^{\circ} \mathrm{C}$. This is not expected, and probably stems from moisture absorbed from the air [14].

The solvents used are dimethylformamide (Aldrich, analytical grade), 1-methyl-2-pyrrolidinone (Janssen Chimica) and dimethylacetamide (Merck, synthesis grade). At $30^{\circ} \mathrm{C}$, the temperature of the thermostated box with the comb-electrodes, these solvents evaporate rather slowly. This slow evaporation is needed, because the final thicknesses obtained are often below $50 \mu \mathrm{m}$. With the slowly evaporating solvents, not only the time needed to solidify the complete polymer solution is delayed, but also significant changes can be observed in the glassy state when the solvents have a relatively large dipole moment or dielectric constant compared to the polymer. Dimethylformamide and dimethylacetamide have a dipole moment of 3.82 and $3.81 \mathrm{D}$, respectively [15]. The dipole moment of 1-methyl-2pyrrolidinone is $4.09 \mathrm{D}$ [16]. All solvents used have a dipole moment larger than the dipole moment of water, which is $1.85 \mathrm{D} \mathrm{[15].} \mathrm{The} \mathrm{dielectric} \mathrm{constants} \mathrm{of} \mathrm{dime-}$ thylformamide, dimethylacetamide and water are 36.7, 37.8 and 78.5 , respectively [17].

In Figs. 4 and 5 the drying of a solution of $10 \mathrm{wt} \%$ polyacrylonitrile (PAN) in dimethylformamide (DMF) is shown. Fig. 4 is the double-logarithmic plot of the measured dielectric permittivity versus time, whereas Fig. 5 gives the corresponding loss factor. A difference in final film thickness is obtained by varying the initial casting thickness, using the same polymer solution. From bottom to top these final thicknesses are respectively $7.5,13$ and $20 \mu \mathrm{m}$. The applied frequency is $1 \mathrm{~Hz}$.

Measurements are started within 1 or 2 min after casting the polymer solution and last for $22 \mathrm{~h}$. The first part of the measurement is not shown because there the impedance of the comb-electrodes is not only determined by the impedance of the polymer solution but also by the resistance within the electrodes. After a certain time dielectric permittivity and loss factor, cal-

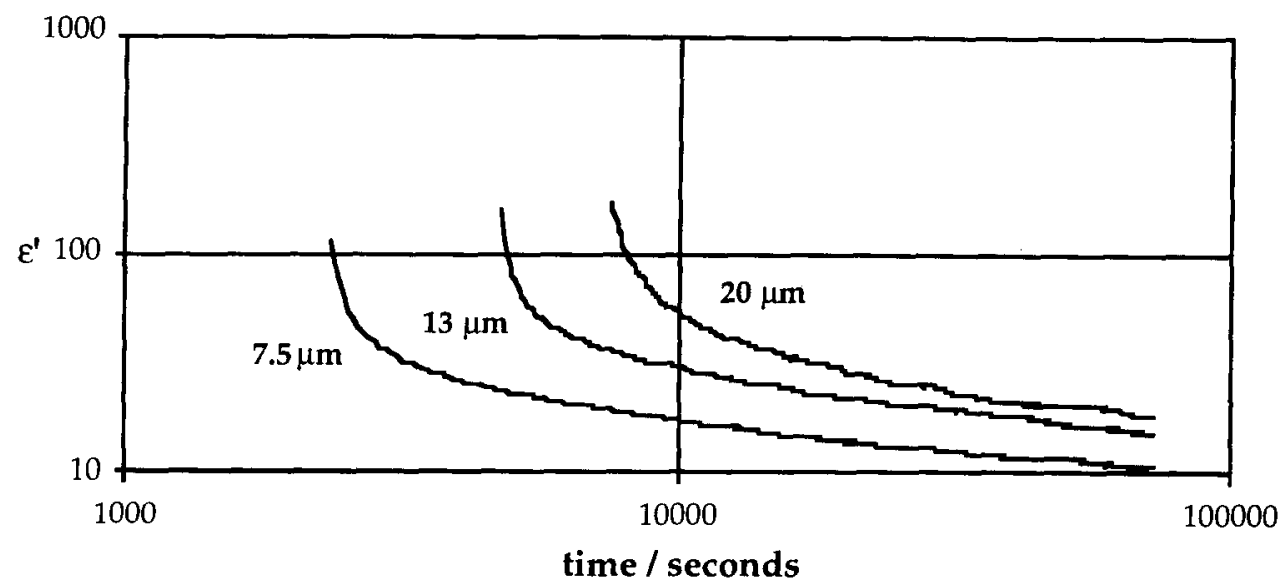

Fig. 4. Dielectric permittivity of a polyacrylonitrile/dimethylformamide solution, as a function of time. Three measurements with dry film thicknesses as indicated are presented. Drying is carried out in a nitrogen atmosphere. The applied frequency is I Hz. 


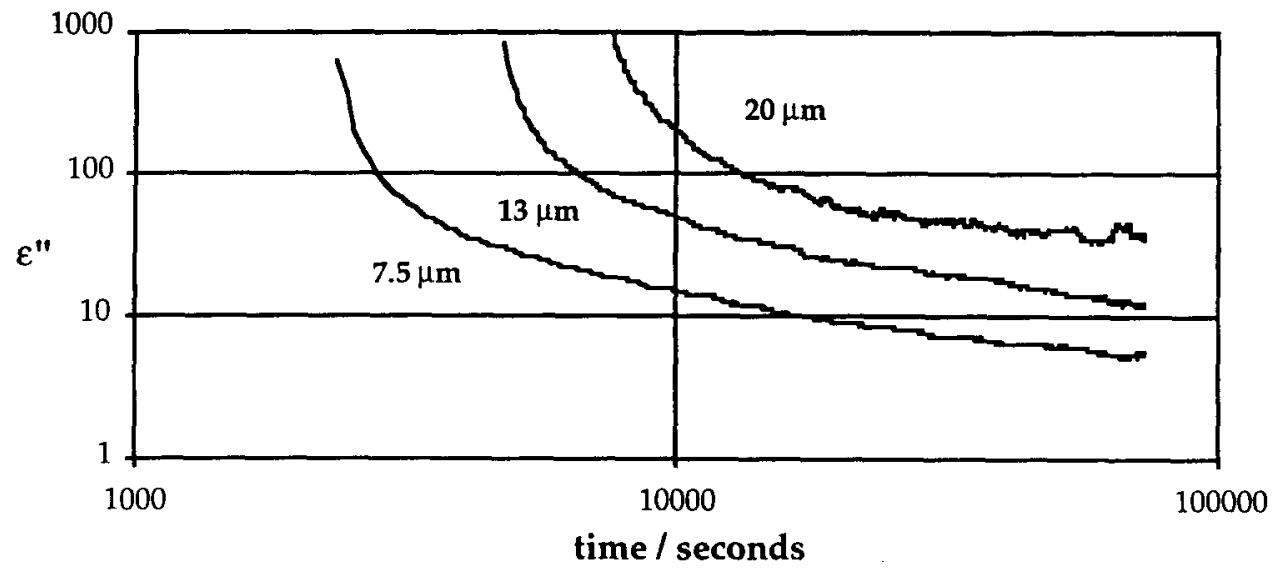

Fig. 5. Loss factor of a polyacrylonitrile/dimethylformamide solution, as a function of time. Three measurements with dry film thicknesses as indicated are presented. Drying is carried out in a nitrogen atmosphere. The applied frequency is $1 \mathrm{~Hz}$.

culated with Eq. (8), can be plotted. A sharp decrease in time is seen in both the dielectric permittivity and loss factor. The high dielectric permittivity, $>100$, is probably due to electrode polarization and the corresponding loss factor due to conduction.

From the frequency dependence of the complex dielectric constant, measured after the presented experiments had stopped, it is concluded that there is no contribution of the conduction of free ions to the measured loss factor. In the final phase of drying the loss factor is equal to the dielectric loss due to the polarization of dipoles.

A region is seen in Figs. 4 and 5 where the curves bend strongly. This "bending point" is attributed to

Table 2

Time needed to vitrify a polymer solution: comparison of dielectric measurements with coagulation of polymer solutions/films in a nonsolvent bath. The initial casting thickness is given between brackets

\begin{tabular}{|c|c|c|c|}
\hline \multirow[t]{2}{*}{ Film thickness $(\mu \mathrm{m})$} & \multirow{2}{*}{$\begin{array}{l}\text { Temperature } \\
\left({ }^{\circ} \mathrm{C}\right)\end{array}$} & \multicolumn{2}{|l|}{ Time to vitrify } \\
\hline & & $\begin{array}{l}\text { Dielectric } \\
\text { experiments } \\
\text { (s) }\end{array}$ & $\begin{array}{l}\text { Coagulation } \\
\text { (s) }\end{array}$ \\
\hline \multicolumn{4}{|c|}{$10 \mathrm{wt} \%$ PAN/DMF solution } \\
\hline $7.5(100)$ & 30 & $2.5 \pm 0.1 \cdot 10^{3}$ & \\
\hline $13(200)$ & 30 & $5.2 \pm 0.2 \cdot 10^{3}$ & \\
\hline $20(300)$ & 30 & $8.8 \pm 1.0 \cdot 10^{3}$ & \\
\hline \multicolumn{4}{|c|}{$10 \mathrm{wt} \%$ PAN/DMF solution } \\
\hline $9 \pm 1(100)$ & $23 \pm 1$ & & $<3.9 \cdot 10^{3}$ \\
\hline $22 \pm 2(200)$ & $24^{+}$ & & $>6.7 \cdot 10^{3}$ \\
\hline $14 \pm 1(200)$ & $24^{+}$ & & $<12.4 \cdot 10^{3}$ \\
\hline
\end{tabular}

the vitrification of the polymer solution or better the vitrification of the solution near the electrodes. Any time after the bending point a polymer film may be lifted from the comb-electrodes and although the films are still plasticized by the solvent they exhibit sufficiently mechanical strength corresponding to the glassy state. The vitrification has been verified by coagulating a cast polymer solution in water, a non-solvent for the polymer. The time between casting the $10 \mathrm{wt} \% \mathrm{PAN} /$ DMF solution and coagulating the remaining film in water has been varied. The underlying idea is that a vitrified, but still plasticized polymer solution, cannot be phase-separated into a polymer-rich and a polymerpoor phase [18]. If the drying time before coagulation is too short the polymer solution can demix and a solid polymer matrix with a porous structure is obtained. The porous structure gives the film an opaque appearance when the pore sizes have a radius comparable to the wavelength of visible light. In case the polymer solution has vitrified, no phase separation can occur upon coagulation in water. The polymer film then remains transparent.

In Table 2 the results of the coagulation experiment are compared to the corresponding times at the bending points in Fig. 4. The bending point is determined by the intersection of the tangents to the left and the right of a curve. For the coagulation experiments several samples with an initial casting thickness of 100 and 200 $\mu \mathrm{m}$ were prepared. The polymer films are coagulated in water at various times after casting the polymer solution. 
The vitrification times from the demixing experiments are noted with reserve. Although several drying times are employed at each casting thickness, one can not exactly give the vitrification time, because the change from opaque to transparent appearance after coagulation is gradual. A film that is hazy after coagulation can become transparent after lifting the polymer film from the water bath.

From Table 2 it is concluded that the bending points in Figs. 4 and 5 represent the vitrification of the polymer solution near the electrodes. The larger the initial casting thickness of the polymer solution, the more time it takes to vitrify the entire polymer solution. This is seen by a shift to the right of the drying curves with increasing casting thickness.

From Figs. 4 and 5 one can conclude that there must be a relevant amount of solvent left in the glassy state. Both the dielectric permittivity and the dielectric loss are decreasing further in time. The decrease in solvent is due to diffusion through the vitrified polymer film followed by the evaporation in the nitrogen atmosphere. In the glassy state the diffusion process is the rate limiting step. The tangent of the curves in Figs. 4 and 5 , in the glassy state, is almost constant, which means that the change in time of the dielectric permit-

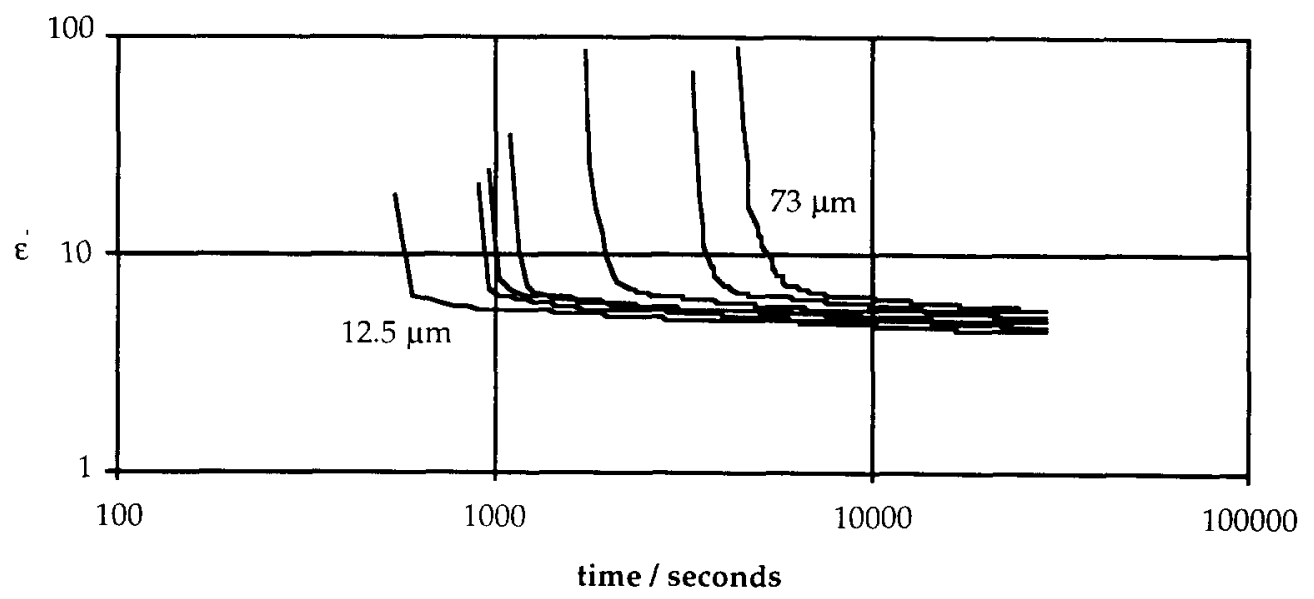

Fig. 6. Dielectric permittivity of a polyethersulfone/dimethylformamide solution, as a function of time. Drying proceeds in a nitrogen atmosphere. The applied frequency is $100 \mathrm{~Hz}$.

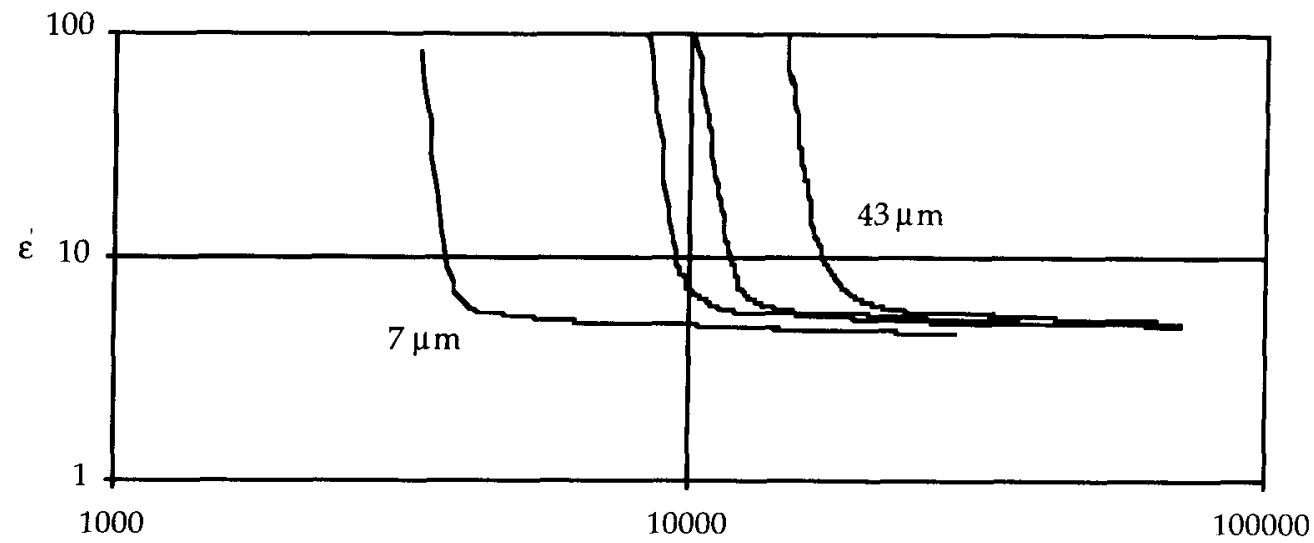

time / seconds

Fig. 7. Dielectric permittivity of a polyethersulfone/1-methyl-2-pyrrolidinone solution as a function of time. Drying proceeds in a nitrogen atmosphere. The applied frequency is $100 \mathrm{~Hz}$. 


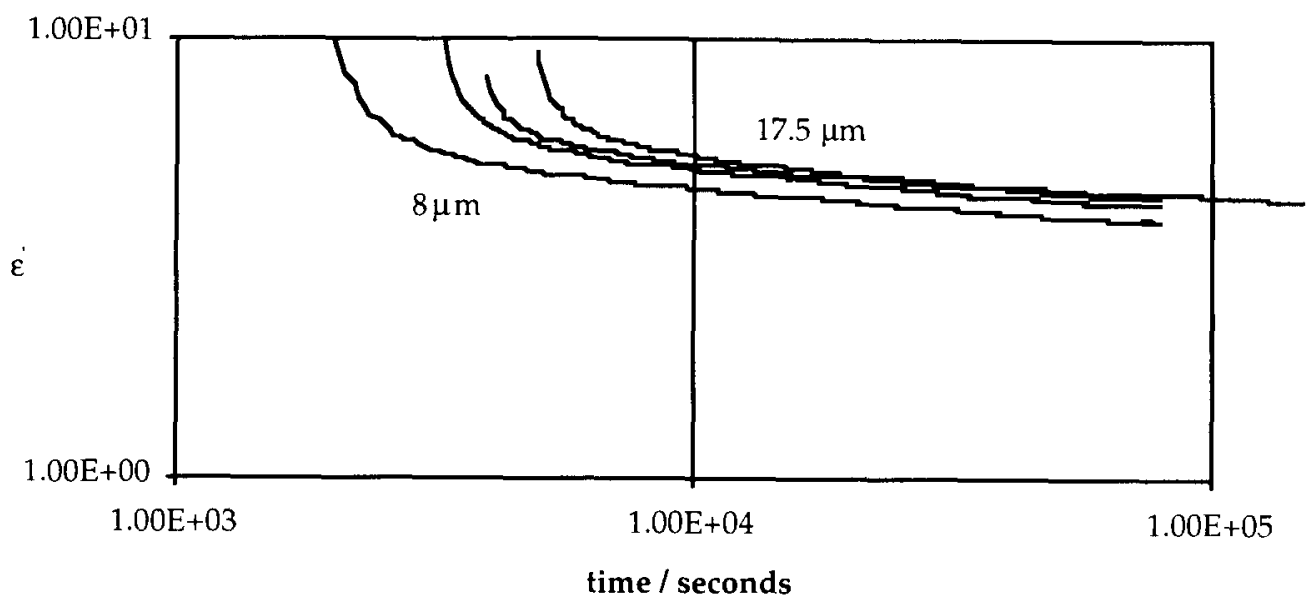

Fig. 8. Dielectric permittivity of a polyimide/dimethylformamide solution as a function of time. Drying proceeds in a nitrogen atmosphere. The applied frequency is $1 \mathrm{~Hz}$.

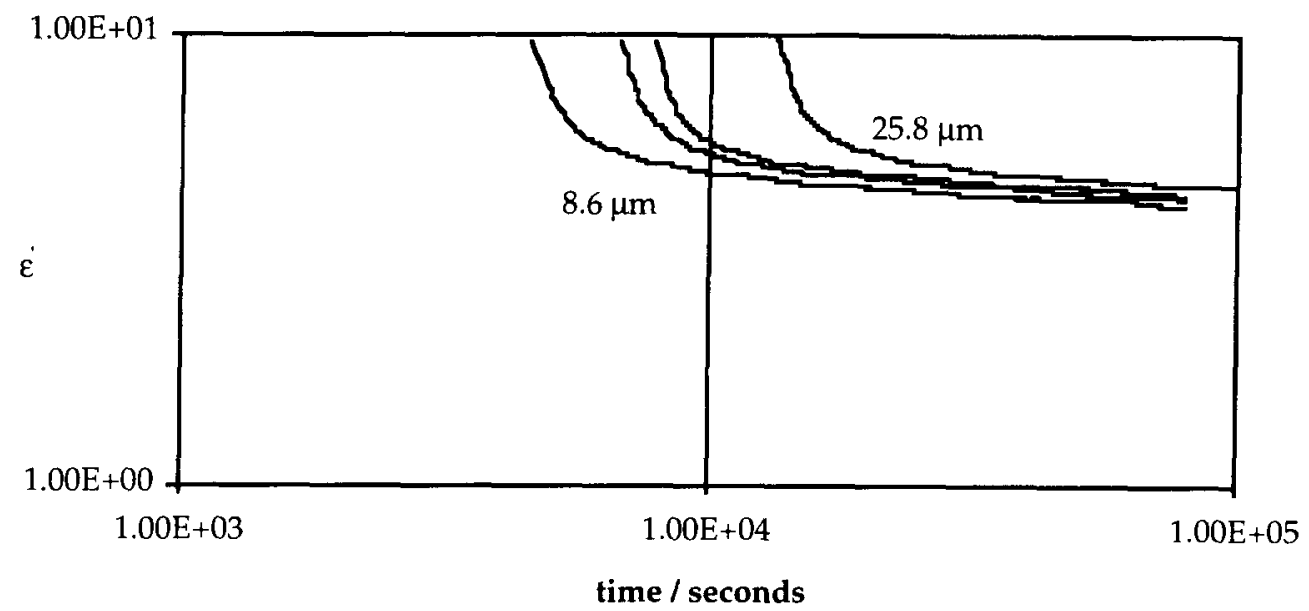

Fig. 9. Dielectric permittivity of a polyimide/dimethylacetamide solution, as a function of time. Drying proceeds in a nitrogen atmosphere. The applied frequency is $1 \mathrm{~Hz}$.

tivity and dielectric loss near the comb-electrodes is approximated by the following relation:

$\epsilon^{\prime}, \epsilon^{\prime \prime} \propto t^{-\prime \prime}$

with $n$ the absolute value of the slope in the doublelogarithmic plots given.

From Eq. (10) it is clear that drying proceeds in a self-decreasing rate.

Both the dielectric permittivity and the dielectric loss show the same trend during drying. In the following experiments only the dielectric permittivity will be shown. After vitrification of the polymer solution the ratio of dielectric loss and permittivity becomess smaller than 0.1 and the accuracy of the dielectric loss is being lost.

In Fig. 6 the dielectric permittivity is given as a function of time, for the drying of a solution of $25 \mathrm{wt} \%$ polyethersulfone (PES) E1000 in DMF.

The final film thicknesses are, from the left to the right, 12.5, 13.5, 16, 19, 31, 51 and $73 \mu \mathrm{m}$. The applied frequency is $100 \mathrm{~Hz}$. Measurements at other frequencies will show the same trend. Every measurement takes $8 \mathrm{~h}$.

In Fig. 7 the drying curve is given for a solution of $30 \mathrm{wt} \%$ PES in 1-methyl-2-pyrrolidinone (NMP). The same frequency of $100 \mathrm{~Hz}$ has been applied. 
The final film thicknesses in Fig. 7 are 7, 20, 29 and $43 \mu \mathrm{m}$. Comparing the time scales of this figure with the former one, it is noticed that a longer time is needed for the vitrification of a solution with NMP as the solvent than with DMF.

In Figs. 8 and 9 the drying of a solution with the polyimide XU 218 is represented. In one case the solvent is DMF and in the other dimethylacetamide (DMAc). The difference between the two solvents is that the latter solvent has one more methyl group, instead of a hydrogen atom, connected to the carbonyl group.

The final film thicknesses in Fig. 8 are, from the left to the right, $8.0,13.5,12.6$ and $17.5 \mu \mathrm{m}$. The frequency is $1 \mathrm{~Hz}$.

The applied frequency in Fig. 9 is the same as in Fig. 8. Final film thicknesses are ranging from 8.6, 14.2, 14.8 to $25.8 \mu \mathrm{m}$. Although there is only a small difference between DMF and DMAc, considerably more time is needed to obtain a glassy film, with the same thickness, from a solution with DMAc. The differences are not that obvious compared to the drying of PES dissolved in DMF or NMP.

From the experiments shown in this section a clear point that is attributed to the vitrification of the polymer solution can be identified. After vitrification, loss of residual solvent is controlled by diffusion of the solvent through the plasticized polymer matrix towards the free surface where evaporation can take place. In the next section this diffusion process will be modelled.

\section{A model for the evaporation step}

The removal of solvent is controlled by the evaporation, diffusion in the gas phase above the cast polymer solution, and diffusion in the polymer solution towards the solution/nitrogen interface where evaporation takes place.

Interest is directed to the final phase of the drying process, i.e. when the glassy state of the polymer is reached. Drying is controlled by diffusion to the nitrogen exposed side of the polymer film. In order to know the absolute solvent flux through the boundary, the solvent diffusion coefficient has to be known as a function of volume fraction, concentration or activity. The dependence of the solvent diffusion coefficient on com- position is not known, and therefore the concentration profile during drying cannot be calculated.

In the literature diffusion coefficients measured with a variety of experimental techniques are presented. Usually stepwise changes of solvent activity are applied to a sample that is at thermodynamic equilibrium. The system under investigation adapts a new equilibrium state with every change of solvent activity in its environment. The process is not instantaneous but kinetically determined.

Determination of the diffusion coefficient in the glassy state can be tedious due to the relatively slow diffusion processes and sometimes it is made impossible by the strong dependence on solvent concentration. The strong solvent dependence is noticed in the case of polymer-solvent systems. Swelling of the polymer matrix will significantly increase the diffusion coefficient. This is in contrast with polymer-penetrant systems, i.e. in which no or negligible swelling is involved and in which diffusion does not depend on solvent activity [19].

In polymer-solvent systems, the diffusion coefficient can even vary over decades within the glassy state $[19,20]$. Often the logarithm of measured diffusion coefficients is plotted as a function of solvent concentration. This strong dependence on the amount of solvent has certain drawbacks. Very often "paired" sorption and desorption curves do not match, indicating that Fick's second law does not hold. Furthermore polymer relaxation may interfere to a certain extent with sorption and desorption experiments [5].

On the basis of the strong dependence of diffusion coefficient $D$ on solvent concentration $c$, one may assume the following solvent concentration profile in the glassy state.

In Fig. 10 the solvent concentration is zero at the nitrogen exposed side of the polymer film, and has a non-zero value at the glass plate side. At the latter interface the derivative of the concentration with respect to the direction $z$ is zero, i.e. no flux occurs through this boundary. The concentration profile must have a steeper gradient near the interface where evaporation takes place compared to steady-state permeation with the same solvent concentrations at both interfaces because in steady-state permeation there is a non-zero gradient for $z=0$ and fluxes are independent of the distance in the film. 


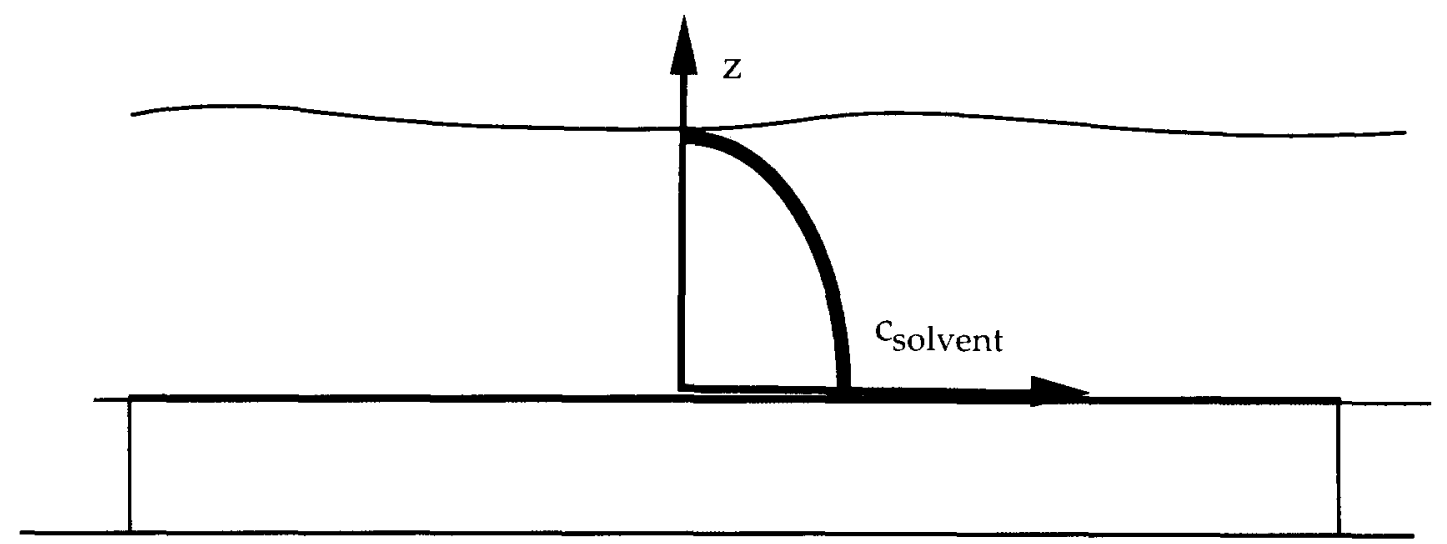

Fig. 10. Schematically drawn concentration profile of solvent in the glassy state

For comparison the concentration profile in steadystate transport is calculated assuming an exponential dependence of the diffusion coefficient on solvent concentration.

$D=D_{0} \exp (\gamma c)$

$z=0: c=c_{0}$

$z=L: c=0$

where $\gamma$ is a so-called plasticization constant and $L$ the total film thickness.

One can readily derive the concentration as a function of distance in the film using Fick's first law.

$c(z)=\frac{1}{\gamma} \ln \left(\frac{z}{L}+\exp \left(\gamma c_{0}\right) \cdot\left(1-\frac{z}{\mathrm{~L}}\right)\right), \gamma>0$

$c(z)=c_{0}\left(1-\frac{z}{L}\right), \gamma=0$

The ratio of the gradients at $z=L$ and $z=0$ is equal to:

$\frac{\partial c}{\partial z_{z-I}} / \frac{\partial c}{\partial z_{z-0}}=\exp \left(\gamma c_{0}\right)=\frac{D\left(c_{0}\right)}{D_{0}}$

The result of Eq. (13) is always larger than 1 if the plasticization constant is not zero. The ratio of the diffusion coefficients at concentration $c_{0}$ and at concentration $c=0$ may even vary over decades for a polymer solution in the glassy state. When Eq. (11) is valid during the drying of a polymer film and there is a zero concentration gradient at $z=0$, then the concentration gradient near the nitrogen exposed side of the film is steeper than the corresponding concentration gradient in steady-state permeation.

For a polymer-solvent system it is likely that the diffusion coefficient strongly depends on the solvent concentration and that during drying of a polymer film in the glassy state the concentration gradient is steep near the nitrogen exposed side of the film. Therefore, Fig. 10 is simplified to Fig. 11.

In Fig. 11 the polymer film is divided into two regions, i.e. a bulk and a skin layer, with thicknesses of $L-\delta$ and $\delta$ respectively. The concentration gradient is completely situated in the skin layer. The gradient in the bulk layer is neglected, although it must be there for else no further drying will happen.

With the assumptions made it is implicitly stated that

$\delta \ll L$

Drying proceeds due to solvent diffusing through the skin layer with an apparent diffusion coefficient $D$ followed by evaporation of the solvent into the nitrogen atmosphere. The solvent flux results in a depletion of solvent, which has to be realised in the bulk layer. These two conditions lead to the following time-dependence of the bulk solvent concentration, in which swelling has been neglected.

$$
\begin{aligned}
c\left(t-t_{\mathrm{ref}}\right)= & c\left(t=t_{\mathrm{ref}}\right) \\
& \cdot \exp \left(-\frac{D}{\delta(L-\delta)}\left(t-t_{\mathrm{ref}}\right)\right)
\end{aligned}
$$




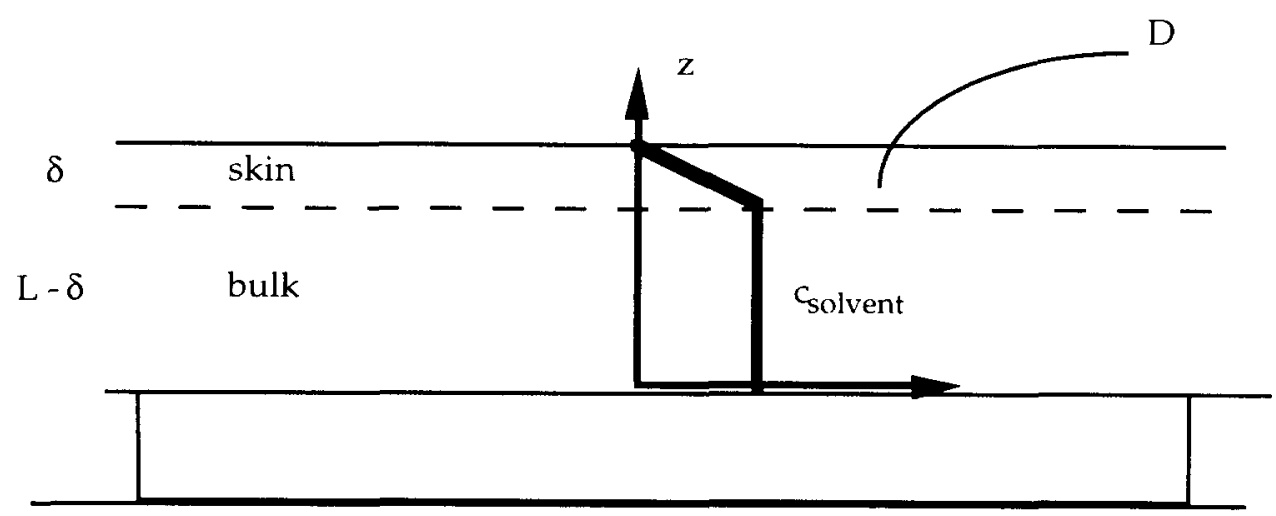

Fig. 11. Simplified schematic drawing showing a solvent concentration profile in the glassy polymer.

From a certain reference time $t_{\text {ref }}$ on, drying proceeds in such a way that the concentration depends only on the concentration, the apparent diffusion coefficient $D$ and thicknesses $\delta$ and $L$ at the reference time. The reference time may be any time, subsequent to the conditions that the polymer film is in the glassy state and inequality (14) is true. During drying the apparent diffusion coefficient and skin and bulk layer thicknesses will vary, and therefore also the time-dependence of the decrease in solvent concentration varies. Both $D$ and $L$ will decrease with time, or better, with the decrease in bulk solvent concentration. In deriving Eq. (15), however, it is assumed that the film thickness is constant.

The solvent concentration and solvent volume fraction are a measure of the amount of solvent per unit volume of polymer film. The concentration $c$ can be related to the dielectric permittivity. The parameter $\epsilon^{\prime}-1$ is a measure of the number of dipoles, multiplied with respective dipole moments, per unit volume. A plot of the ratio of dielectric loss and the measured dielectric permittivity subtracted with the permittivity of the pure polymer versus time is constant in the glassy polymer. It confirms the idea that the decrease of dielectric permittivity and dielectric loss in time is directly related to the amount of solvent. Neglecting both swelling of the polymer film and enhanced mobility of the polymer chains, due to the presence of solvent, the following relation between solvent concentration, and dielectric permittivity of the plasticized and dry polymer film, is established.

$c(t) \propto \epsilon^{\prime}(t)-\epsilon_{\text {polymer }}^{\prime}$
The same relation is used by Day [21] in the determination of water diffusion coefficients in polyimides and is based on the large dielectric constant of water, i.e. $78.5[15,17]$, compared to the permittivity of the polymer. Although the dielectric constants of the solvents used are not as large as the dielectric constant of water, they are still large compared to the polymers. Combining Eqs. (15) and (16) gives:

$$
\begin{aligned}
\epsilon^{\prime}\left(t-t_{\mathrm{ref}}\right)-\epsilon_{\mathrm{p}}^{\prime}= & \left(\epsilon^{\prime}\left(t=t_{\mathrm{ref}}\right)-\epsilon_{\mathrm{p}}^{\prime}\right) \\
& \cdot \exp \left(-\frac{D}{\delta(L-\delta)}\left(t-t_{\mathrm{rel}}\right)\right)
\end{aligned}
$$

Plotting the measured dielectric permittivity minus the dielectric permittivity of the pure film, as a function of time, one can evaluate Eq. (17). One may take any reference time $t_{\text {ref }}$, and draw a tangent to the curve at $t_{\text {ref }}$. The tangent line will intersect the time axis, and the reciprocal of the time-span $\theta$ between the intersection and $t_{\text {ref }}$ equals:

$\frac{1}{\theta}=\frac{D}{\delta(L-\delta)}$

Multiplying the reciprocal of time $\theta$ with the thickness $L$ of the dry polymer film one obtains the solvent mass transfer coefficient $k$ if inequality (14) holds and swelling may be neglected.

$\frac{L}{\theta}=\frac{D L}{\delta(L-\delta)} \cong k$

Multiplying the reciprocal of time $\theta$ with the square of thickness $L$, gives Eq. (20). 


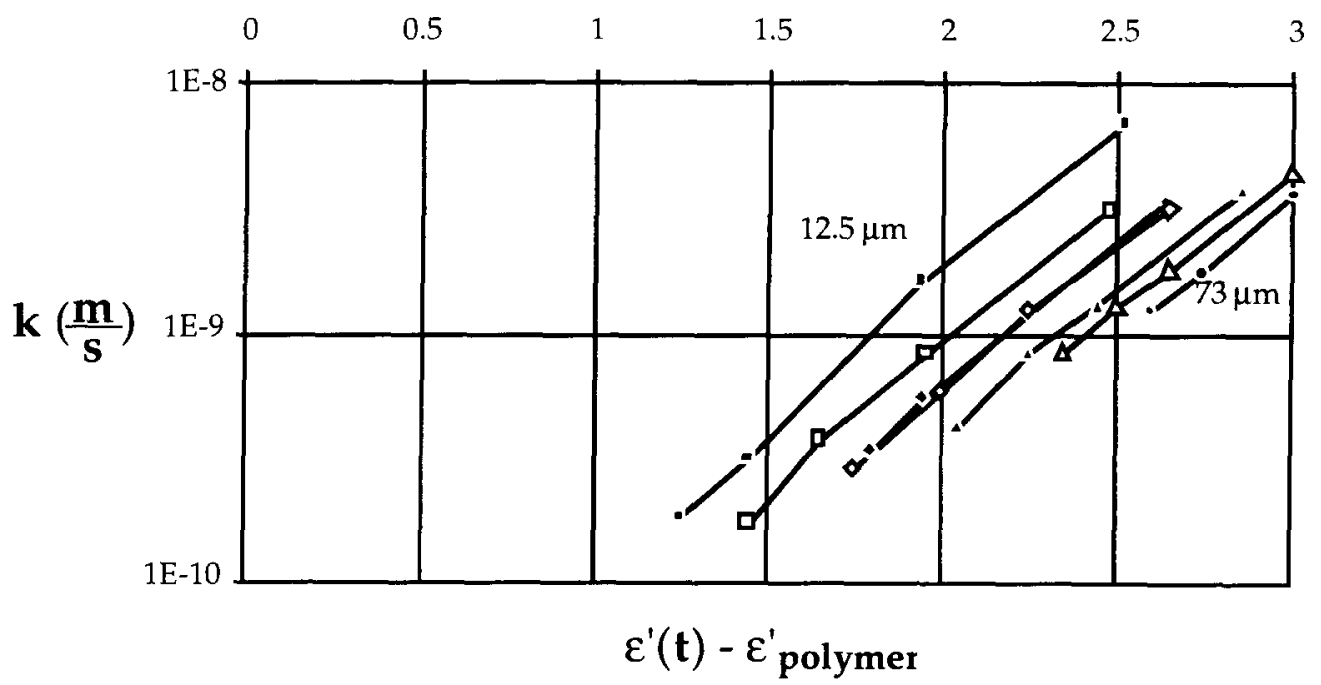

Fig. 12. Mass transfer coefficient of dimethylformamide in a polyethersulfone film as a function of the measured dielectric permittivity minus the permittivity of the polymer.

$\frac{L^{2}}{\theta}=\frac{D L^{2}}{\delta(L-\delta)}=\frac{D}{x(1-x)} \cong \frac{D}{x}$

with $x=\delta L^{-1}$.

The analysis of dielectric data with the proposed bulk-skin model is performed only when the polymer film is in the glassy state. The conduction of free ions does not contribute then to the losss factor and the dielectric permittivity is not influenced by electrode polarization. Furthermore, inequality (14) is expected to be true for polymer-solvent combinations. The effective layer thickness in which the dielectric properties are measured is $7 \mu \mathrm{m}$ and when film thicknesses exceed $\approx 8 \mu \mathrm{m}$ the concentration gradient in the layer of $7 \mu \mathrm{m}$ covering the electrodes can safely be neglected. The measured dielectric properties are then a measure of solvent concentration.

In the glassy state drying is controlled by the diffusion of solvent through the polymer film towards the nitrogen atmosphere. Therefore it is expected that the solvent concentration profiles in the glassy state are the same for all film thicknesses and can be drawn relative to the nitrogen exposed side and the glass plate side of the film. It is expected that solvent mass transfer coefficients in the glassy state increase with decreasing film thickness due to the decrease in the skin layer thickness.

In Figs. 12 and 13, the mass transfer coefficients of DMF and NMP, respectively, are given as a function of the measured dielectric permittivity minus the permittivity of the pure polymer. The latter value has been taken from Table 1. The $x$-axis is a measure for the bulk solvent concentration or volume fraction. The data has been obtained by application of the aforementioned procedure on Figs. 6 and 7.

The mass transfer coefficient is decreasing with decreasing concentration. The origin is two-fold due to the composite nature of the mass transfer coefficient incorporating an apparent diffusion coefficient and a thickness. First the ratio of the diffusion coefficients at the nitrogen exposed side and glass plate side will decrease during drying. The transition from skin to bulk layer will become less distinct, resulting in a thicker skin layer. Second the diffusion coefficient will decrease throughout the whole film, and therefore also the apparent diffusion coefficient of the skin layer decreases.

The expected thickness dependence of the mass transfer coefficient is shown. A thinner film has a higher solvent mass transfer coefficient at the same concentration or dielectric permittivity at the glass plate side. This is seen most prominent in the experiments with DMF. For NMP the mass transfer coefficient seems to be relatively independent of the film thickness.

The drying process of the polyimide films has been analyzed in the same way. Figs. 14 and 15 give the mass transfer coefficients in XU 218 of dimethylfor- 


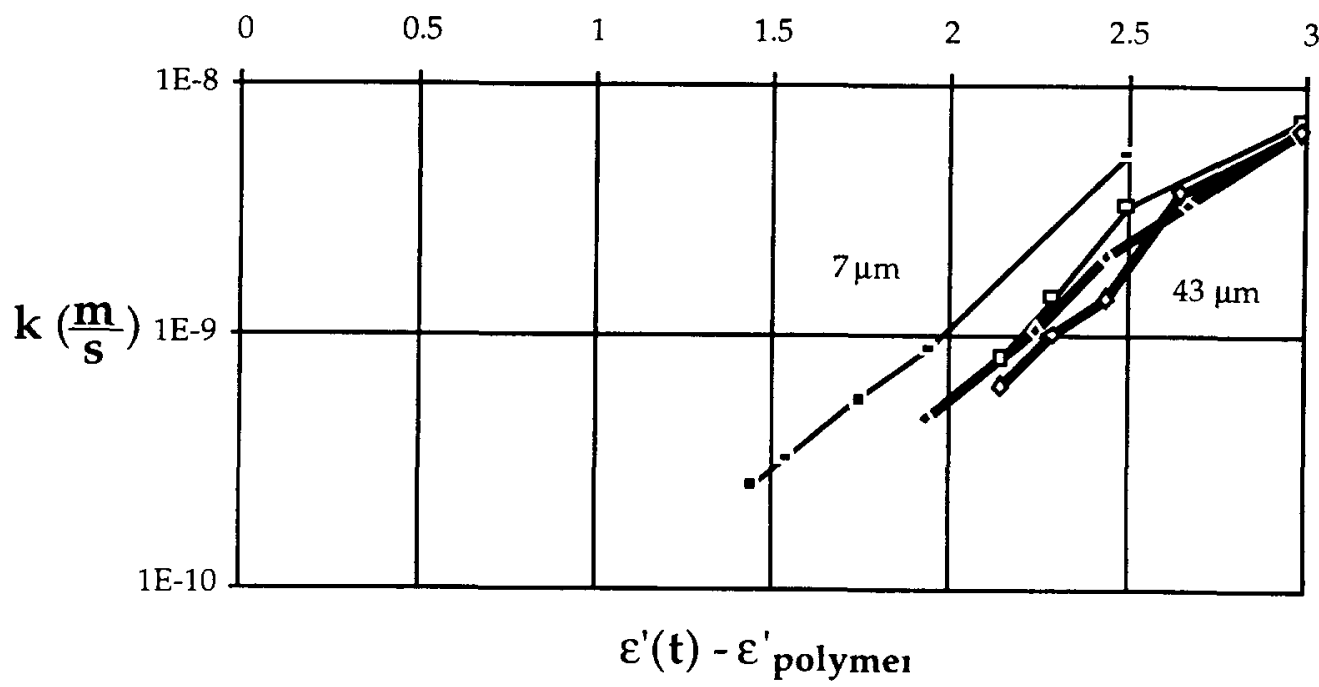

Fig. 13. Mass transfer coefficient of 1-methyl-2-pyrrolidinone in a polyethersulfone film as a function of the measured dielectric permittivity minus the permittivity of the polymer.

mamide and dimethylacetamide, respectively. The dielectric permittivity of the pure polymer is assumed to be 3.0. The deviating values in Table 1 are probably the result of moisture uptake. Fig. 14 represents the mass transfer coefficient of DMF in the polyimide XU 218 , as a function of the "concentration". The same trend is seen: the smaller the final film thickness the higher is the mass transfer coefficient.
In Fig. 15 the DMAc mass transfer coefficient in XU 218 is given, for the measurement data presented in Fig. 9. No strong dependence of the mass transfer coefficient on the polyimide film thickness is noticed.

From Figs. 12 to 15 it is clear that the solvent mass transfer coeffcient is a function of the film thickness. With DMF as a solvent, the increase of the mass transfer coefficient with decreasing film thickness is more pro-

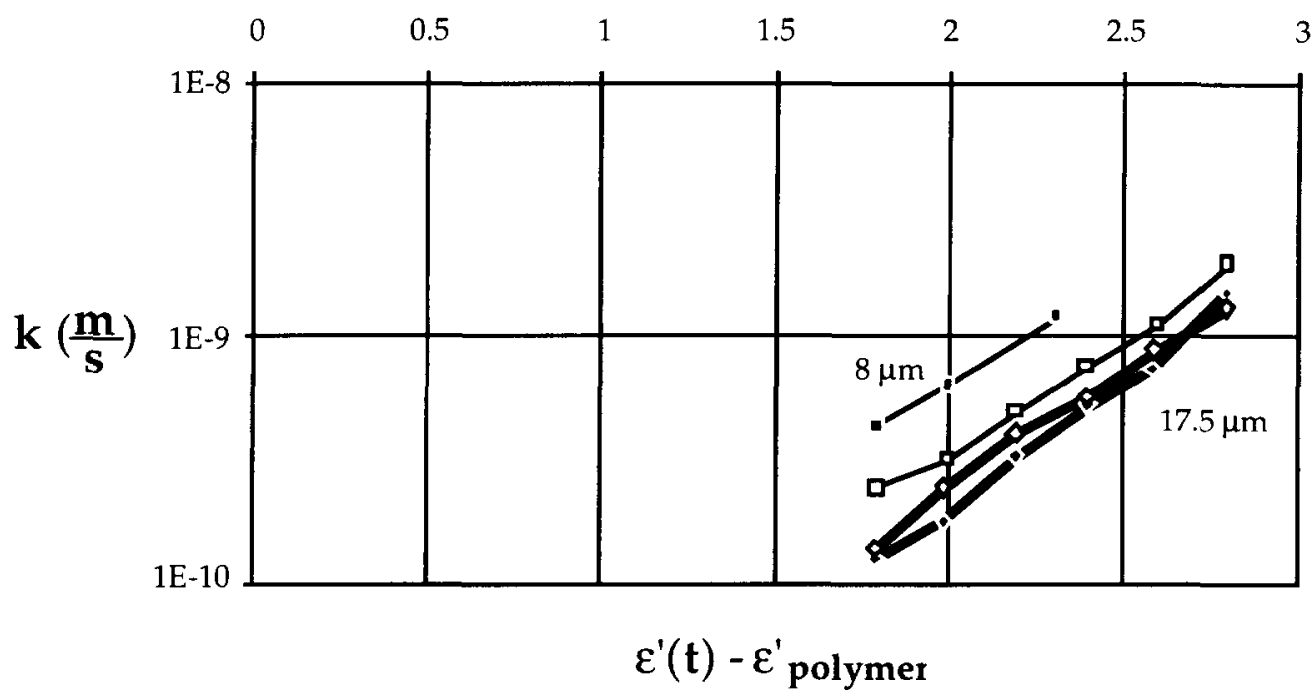

Fig. 14. Mass transfer coefficient of dimethylformamide in a polyimide XU 218 film as a function of the measured dielectric permittivity minus the permittivity of the polymer. 


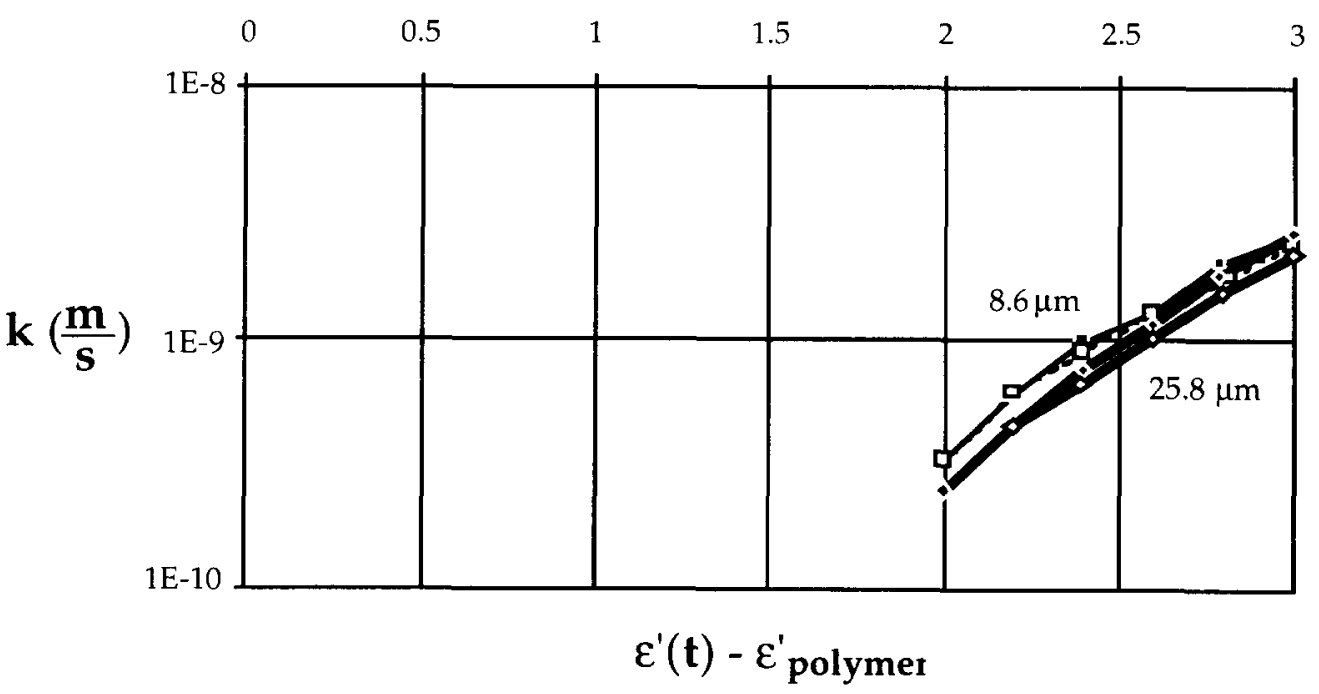

Fig. 15. Mass transfer coefficient of dimethylacetamide in a polyimide XU 218 film as a function of the measured dielectric permittivity minus the permittivity of the polymer.

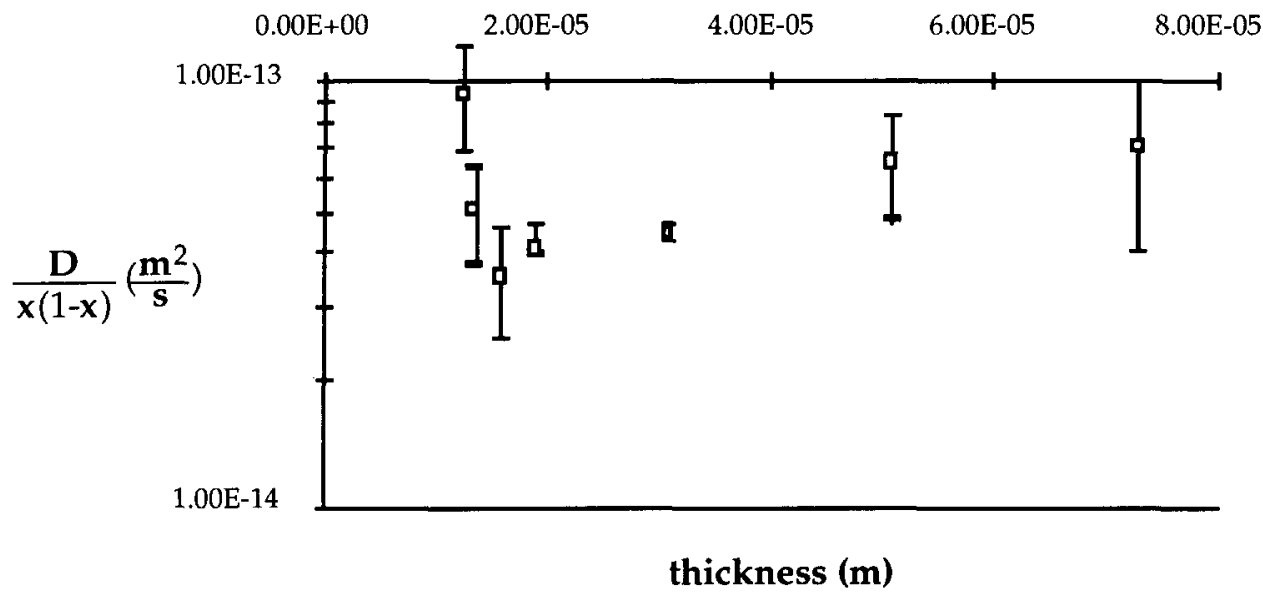

Fig. 16. $D x^{-1}(1-x)^{-1}$ versus film thickness for dimethylformamide evaporating from polyethersulfone. Values are taken at a fixed concentration, i.e. measured dielectric permittivity minus permittivity of the pure polymer is 2.5 . The errors in $D x^{-1}(1-x)^{-1}$ result from the error in the film thickness.

nounced than with NMP and DMAc, the more slowly evaporating solvents. If the solvent concentration profile in the polymer film could be drawn relative to the nitrogen exposed side and the glass plate side, then the parameter $D x^{-1}(1-x)^{-1}$ in Eq. (20) would not be a function of the film thickness. The dimensionless number $x=\delta L^{-1}$ would then be the same for all film thicknesses, and the same apparent diffusion coefficient $D$ could be assigned to the skin layer. For the solvents NMP and DMAc the parameter $D x^{-1}(1-x)^{-1}$ is slightly decreasing with decreasing film thickness.
With the solvent DMF a slight decrease is noticed when decreasing the film thickness down to $15 \mu \mathrm{m}$, followed by a rather sharp increase in $D x^{-1}(1-x)^{-1}$ when further decreasing the film thickness. This is seen with both polyethersulfone and polyimide. In Fig. 16 the variation of $D x^{-1}(1-x)^{-1}$ with film thickness is shown for PES/DMF, at a fixed concentration.

As the parameter $D x^{-1}(1-x)^{-1}$ is not a constant value for all film thickness, one might conclude that during the evaporation of residual DMF from thin films the polymer film is not in its equilibrium state. The 
evaporation is too fast compared to the volume relaxation that has to be realized because of the evaporation.

The ratio $x$ of skin layer thickness and total film thickness is (much) smaller than 1. The apparent diffusion coefficient $D$ in the skin layer is therefore (much) smaller than the depicted values of $D x^{-1}(1-x)^{-1}$, which in the experiments presented range from $10^{-15}$ to $10^{-12} \mathrm{~m}^{2} \mathrm{~s}^{-1}$.

\section{Discussion and conclusions}

With the comb-electrodes a technique has been found that can monitor the drying of a polymer solution without disturbing the drying process. An advantage of this technique is that the polymer solution is supported and is prevented from flowing. Furthermore the accuracy of the measurements is no function of the sample dimensions. The only restrictions in applying Eq. (8), the direct relation between the measured capacity and the dielectric permittivity of the polymer film, are that film thicknesses should exceed $7 \mu \mathrm{m}$ and the impedance of the material in between the electrodes must be much larger than the impedance in the electrode configuration. Due to the internal resistance of the comb-electrodes it is only the final stage of drying that can be followed. A clear transition is noticed on a double-logarithmic plot of dielectric permittivity or loss versus time. The transition is attributed to the vitrification of the polymer solution near the comb-electrodes. After vitrification drying proceeds at a self-decreasing rate. The rate at which the solvent concentration decreases depends on the diffusion coefficient of the solvent, which in turn depends on the solvent concentration. This circular argument applies, at any time, in the glassy state of a polymer. The powerlaw coefficient $n$ in Eq. (10), determined from the dielectric loss, varies for the polyacrylonitrile/DMF film from 0.5 to 0.7 . Except for the shown experiments with an applied frequency of $100 \mathrm{~Hz}$, other experiments with polyethersulfone have been performed at 10 and $1000 \mathrm{~Hz}$. The power-law coefficient for the polyethersulfone films with DMF and NMP varies from 0.4 to 0.7 , irrespective of the applied frequency.

The kinetics of the drying process have been modelled in a simple way. Although no information is obtained about the diffusion coefficients involved one can estimate the mass transfer coefficients. In the model swelling is neglected and the concentration gradient needed for evaporation is situated in a relatively thin layer near the evaporation surface. The mass transfer coefficients are decreasing with bulk solvent concentration. Calculated mass transfer coefficients range from $10^{-10}$ to $10^{-8} \mathrm{~m} \mathrm{~s}^{-1}$. These values are lower, as expected, than mass transfer coefficients for liquids in porous media, which roughly vary between $10^{-7}$ to $10^{-4} \mathrm{~m} \mathrm{~s}^{-1}$ [22]. Although the calculated solvent mass transfer coefficients in the glassy state can not be compared with values from literature, their order of magnitude can be estimated when knowing the diffusion coefficient and skin layer thickness. The solvents applied in the dielectric experiments are expected to have a diffusion coefficient of the order $10^{-14}$ to $10^{-15}$ $\mathrm{m}^{2} \mathrm{~s}^{-1}$ or even less when their concentration in glassy polymers approaches zero. The skin layer thickness is smaller than the dry film thickness. Assuming that the skin layer thickness is of the order 0.1 to $1 \mu \mathrm{m}$, then solvent mass transfer coefficients are estimated to be of the order $10^{-9}$ to $10^{-7} \mathrm{~m} \mathrm{~s}^{-1}$ which compares well with the mass transfer coefficients calculated from the dielectric experiments.

Due to the large resistance to diffusion at the evaporation interface, one might expect that the concentration profile can be drawn relative to the nitrogen exposed side and the glass plate side, i.e. as a function of the relative height in the polymer film. This idea is rejected by the observation that for the polymer films with DMF the parameter $D x^{-1}(1-x)^{-1}$ of Eq. (20) is strongly increasing with decreasing film thickness, below $15 \mathrm{~mm}$. With thin films it is very likely that excess free volume is frozen into the glassy state. Vrentas et al. [5] compare the characteristic relaxation time of a material, which is in the glassy state of the order of one hour or more, with a characteristic time of diffusion. When the latter time is small compared to the time of volume relaxation excess free volume is frozen into the polymer material. A global estimate of the characteristic time of diffusion or drying would be given by the ratio of the film thickness and the calculated solvent mass transfer coefficient. The mass transfer coefficient is a measure of the rate of drying and the film thickness is a measure of the volume in which drying has to be realized. With film thicknesses of the order $10 \mathrm{~mm}$ and calculated solvent mass transfer coefficients of the order $10^{-9} \mathrm{~m} \mathrm{~s}^{-1}$, the characteristic time of drying is about $10000 \mathrm{~s}$. The relaxation times in 
glassy polymers strongly depend on the solvent content and the temperature relative to the corresponding glass transition temperature [23]. From the WLF equation it can be seen that for all polymers in the glassy state a decrease in temperature of only $3^{\circ} \mathrm{C}$ will theoretically result in about a tenfold increase of the relaxation time [23]. For polyethersulfone E1000 and polyimide XU 218 the glass transition temperatures are ca. 210 and $280^{\circ} \mathrm{C}$, respectively (data supplied by manufacturers). Although the differences between the temperature of the experiment, which is $30^{\circ} \mathrm{C}$, and the glass transition temperatures are so large that the WLF equation does not hold anymore, one can conclude that the characteristic time of diffusion might well become smaller than the time corresponding to volume relaxation. This does explain then the increase of the parameter $D x^{-1}(1-x)^{-1}$ with decreasing polymer film thickness, because the polymer matrix does not respond to changes in solvent concentration and diffusion is enhanced in the polymer which contains an excess of free volume.

Experimentally it has been observed that the drying of polymer solution may proceed too fast compared the compaction of the polymer matrix. This effect strongly depends on the film thickness because the characteristic time of diffusion is proportional to the square of film thickness, and the polymer relaxation time is no function of the film thickness at all.

In the present paper a novel approach is used to monitor the drying of a solvent-cast polymer film. In the vitrified state drying proceeds at a self-decreasing rate. Solvent mass transfer coefficients in the glassy state are calculated from the dielectric data. It has been found experimentally that when the film thickness decreases too much volume relaxation proceeds slowly compared to the drying process and excess free volume is frozen into the polymer film.

\section{List of symbols}

a surface

c solvent concentration in polymer film

$g$ response of dielectric displacement to unit step in electric field

$\begin{array}{ll}i & \text { imaginary unit } \\ k & \text { geometry factor or solvent mass transfer }\end{array}$ coefficient $n \quad$ slope in double-logarithmic plot of dielectric permittivity or loss versus time

$t$ time

$z \quad$ place coordinate

e basic vector of Carthesian system

n outward normal on surface

$\gamma \quad$ plasticization constant of the diffusion coefficient

$\delta \quad$ skin layer thickness

$\epsilon_{0}$ dielectric permittivity of free space

$\epsilon^{*} \quad$ complex dielectric constant

$\epsilon^{\prime}$ dielectric permittivity

$\epsilon^{\prime \prime} \quad$ dielectric loss

$\theta$ time-span

$\rho$ resistivity

$\omega$ angular frequency

$C^{*}$ complex capacity

A cross-section of one comb electrode perpendicular to current through the electrode

$D$ diffusion coefficient

I current

$L \quad$ film thickness or length of a needle of comb electrode

$Q \quad$ charge

$R \quad$ resistance

$S$ surface

$\mathrm{V}$ potential, voltage

Z impedance

D electric displacement

$\boldsymbol{E}$ electric field

$\boldsymbol{P}$ polarization

\section{Acknowledgements}

A. Kooy of the MESA-institute of the University of Twente is acknowledged for making the combelectrodes.

\section{References}

[1] L.C.E. Struik, Physical Aging in Amorphous Polymers and Other Materials, Elsevier, Amsterdam, 1978.

[2] G.S. Park, The glassy state and slow process anomalies, in J. Crank and G.S. Park (Eds.), Diffusion in Polymers, Academic Press, New York, 1968.

[3] W.R. Vieth, Diffusion in and Through Polymers, Principles and Applications, Hanser, 1991. 
[4] J.W.F. Spitzen, G.H. Koops, M.H.V. Mulder and C.A. Smolders, The influence of membrane thickness on pervaporation performance, in R. Bakish (Ed.), Proc. 3rd Int. Conf. Pervaporation Processes Chem. Ind., Nancy, France, 1988.

| 5 | J.S. Vrentas, C.M. Jarzebski and J.L. Duda, A Deborah number for diffusion in polymer-solvent systems, AIChE J., 21 ( 1975) 894-901.

|6| M.E. Rezac, P.H. Pfromm, L.M. Costello and W.J. Koros, Aging of thin polyimide-ceramic and polycarbonate-ceramic composite membranes, Ind. Eng. Chem. Res., 32 (1993) 19211926.

17| L.A. Dissado, R.M. Hill and T.A. Strivens, Power-law decay of conductance during drying of latex paints, J. Phys. D, Appl. Phys., 22 (1989) 713-716.

|8| R.M. Hill, L.A. Dissado and T.A. Strivens, Applications of dielectric measurements to studies of latex film formation, in A.V. Patsis (Ed.), 15th Int. Conf. Org. Coatings Sci. Technol., 1989, pp. 71-86.

$19 \mid$ S.D. Senturia and N.F. Sheppard, Jr., Dielectric analysis of thermoset cure, Adv. Polym. Sci., 80 ( 1986) 1-47.

| $10 \mid$ S.D. Senturia and S.L. Garverick, Methods and apparatus for microdielectrometry, US Patent, 4423371 (1983).

111 | N.F. Sheppard, D.R. Day, H.L. Lee and S.D. Senturia, Microdielectrometry, Sensors Actuators, 2 (1982) 263-274.
[12] H. Markovitz, Boltzmann and his superposition principle: centenary observance, Proc. VIIth Int. Congr. Rheology, 1976, pp. 37-39.

[13] G. Chiodelli and P. Lupotto, Experimental approach to the impedance spectroscopy technique, J. Electrochem. Soc., 138 (1991) 2703-2710.

[14] R.K. Brunner, Ciba-Geigy, private communication

[15] Handbook of Chemistry and Physics, CRC, Cleveland, $\mathrm{OH}$.

[16] T.E. Daubert and R.P. Danner, Physical and Thermodynamic Properties of Pure Chemicals, Data Compilation, Taylor and Francis, 1993.

[17] Bruker Almanac, 1993.

[18] S.-G. Li, Preparation of hollow fiber membranes for gas separation, Ph.D. Thesis, University of Twente, Enschede, The Netherlands, 1988.

[19] H. Fujita, Diffusion in polymer-diluent systems, Fortschr. Hochpolym.-Forsch., 3 (1961) 1-47.

[20] C.M. Hansen, A mathematical description of film drying by solvent evaporation, J. Oil Col. Chem. Assoc., 51 (1968) $27-$ 43.

[21] D.R. Day, Moisture monitoring at the $\mathrm{PI}-\mathrm{SiO}_{2}$ interface using microdielectric sensors, in C. Feger, M.M. Khojasteh and J.E McGrath (Eds.), Polyimides: Materials, Chemistry and Characterization, Elsevier, Amsterdam, 1989.

[22] J.A. Wesselingh and R. Krishna, Mass Transfer, Ellis Horwood, 1990, Chap. IV.

[23] J.D. Ferry, Viscoelastic Properties of Polymers, Wiley, New York, 1980, Chap. XI. 